\title{
Efficacy of levofloxacin on macrolide-unresponsive and corticosteroid-resistant refractory Mycoplasma pneumoniae pneumonia in children
}

\author{
Miaomiao Lin ${ }^{1}$, Lifeng Shi ${ }^{2}$ Airong Huang ${ }^{1}$, Dongshi Liang ${ }^{1}$, Lisha Ge ${ }^{1}$, Yimei Jin ${ }^{1}$ \\ ${ }^{1}$ Department of Pediatrics, The Second Affiliated Hospital of Wenzhou Medical University, WenZhou 325027, China; ${ }^{2}$ Department of Pediatrics, \\ The Yueqing Affiliated Hospital of Wenzhou Medical University 325600, WenZhou, China \\ Contributions: (I) Conception and design: M Lin, Y Jin; (II) Administrative support: Y Jin; (III) Provision of study materials or patients: M Lin, L Shi, \\ A Huang, D Liang; (IV) Collection and assembly of data: M Lin, L Ge; (V) Data analysis and interpretation: M Lin; (VI) Manuscript writing: All \\ authors; (VII) Final approval of manuscript: All authors. \\ Correspondence to: Yimei Jin. Department of Pediatrics, The Second Affiliated Hospital of Wenzhou Medical University, WenZhou 325027, China. \\ Email: 69365560@qq.com.
}

\begin{abstract}
Background: This study aimed to summarize the experience in treatment of Mycoplasma pneumoniae pneumonia (MPP) with worsening lung shadow despite treatment with appropriate antimicrobials and corticosteroid in children.

Methods: All patients satisfied refractory MP pneumonia (RMPP) diagnostic criteria were enrolled. The clinical manifestations, laboratory findings, imaging features, treatments, and outcomes were retrospectively reviewed.

Results: Six patients with an average age of $7.83 \pm 3.13$ years old were included in this study. All the patients were non-responsive to macrolide (ML) and glucocorticoids treatment shown by aggravated clinical symptoms and chest radiographies. The average total duration of fever was $19.5 \pm 8.34$ days and the average time before levofloxacin (LVX) therapy was 10 2.97 days. After LVX treatment, the time of fever was from 1 to 3 days in five cases and 11 days in one case. The MP-DNA copies in the sputum decreased slowly after ML treatment in six patients, while they decreased quickly after LVX treatment in 5 children. A2063G mutation of domain V of $23 S r R N A$ gene was found in five cases. Five patients recovered completely 16-32 days after treatment. One patient developed acute disseminated encephalomyelitis (ADEM) with abnormal brain magnetic resonance imaging and occurred serious sequelae.

Conclusion: The sputum MP-DNA copies and clinical symptoms have a positive correlation with therapeutic efficacy. LVX may be beneficial in treatment of ML-unresponsive and corticosteroid-resistant RMPP in children. RMPP can be gradually cured by effective treatment of LVX, while which can damage the nervous system and lead to severe complications once MP invades brain tissues.
\end{abstract}

Keywords: Refractory Mycoplasma pneumoniae pneumonia (RMPP); macrolide levofloxacin (ML)-unresponsive; corticosteroid-resistant; levofloxacin (LVX)

Submitted May 08, 2019. Accepted for publication Sep 24, 2019.

doi: 10.21037/apm.2019.10.05

View this article at: http://dx.doi.org/10.21037/apm.2019.10.05

\section{Introduction}

Mycoplasma pneumoniae (MP) is the major bacterial pathogen of airways which causes acute and chronic infections of the respiratory tract and accounts for approximately $10-40 \%$ of all lower respiratory tract infections including community- acquired pneumonia (CAP) (1). Azithromycin has been believed to exhibit satisfactory effects in the treatment of Mycoplasma pneumoniae pneumonia (MPP) in the past (2). However, there are still some cases showing clinical and radiological deterioration despite of macrolide (ML) 
antibiotic therapy for 7 days or longer (3) to be defined as refractory Mycoplasma pneumoniae pneumonia (RMPP). Recently, with the rapid increase in the macrolide-resistant Mycoplasma pneumoniae pneumonia (MRMPP), in vitro antimicrobial susceptibility testing showed that MP in most cases was significantly resistant to MLs but sensitive to tetracyclines and fluoroquinolones (4).

Japanese investigators have shown that minocycline can be used in children aged $\geq 8$ years old, and fluoroquinolones can be applied in children aged $<8$ years old when $\mathrm{ML}$ treatment is ineffective in MRMPP (5). Many studies have reported (6-8) that fluoroquinolones [such as ciprofloxacin, moxifloxacin and levofloxacin (LVX)] in combination with glucocorticoid are effective in the treatment of MRMPP and RMPP in children. In this study, we retrospectively reviewed the clinical manifestations, laboratory findings, imaging features, treatments, and outcomes in six children who were treated with LVX after non-responses to MLs, glucocorticoid, and immunoglobulin.

\section{Methods}

\section{Children' data}

A retrospective medical chart review was performed. Clinical features, laboratory data, radiological findings, hospital course (hospital stays and hospitalization expenses), complications, and treatments were recorded. A total of six children were hospitalized in the Department of Pediatrics of Yuying Children Hospital of Wenzhou Medical University from December 2009 to April 2018. All patients satisfied the RMPP diagnostic criteria.

\section{Diagnosis of MPP and RMPP}

MPP can be diagnosed by using an enzyme immunoassay. An acute infection is indicated by the detection of immunoglobulin (Ig) IgM. MP-IgM antibodies $>1: 160$ or a four-fold rise in IgG titer indicated MPP in pediatric patients with pneumonia (9). Polymerase chain reaction (PCR) analysis can also be used to detect MP in sputum and nasopharyngeal or throat swabs. RMPP presents with clinical and radiological deterioration despite ML antibiotic therapy for $\geq 7$ days (10).

\section{Experimental methods}

ELISA was used to measure MP-antibodies, including $\mathrm{IgG}$ and IgM. Real-time fluorescent quantitative PCR was adopted to measure the MP-DNA load at different time points, and the threshold for positivity was $2.5 \times 10^{3}$ genomic DNA copies per $\mathrm{ml}$ of sputum specimens. The sputum was collected before and after ML and LVX therapies as well as after discharge. Amplification of domain V of $23 \mathrm{~S}$ rRNA gene was performed by nested PCR, and then PCR products (including the reference strain) were sequenced (Sangon Biotech Co., Ltd., Shanghai, China). The DNA sequences were compared with that of MP strain $\mathrm{FH}$ (GenBank accession no. X68842) by BLAST, and then the resistance gene mutation was identified.

\section{Results}

\section{Baseline characteristics and clinical manifestations}

A total of six patients were recruited in this study. There were three males and three females with an average age of $7.83 \pm 3.13$ years old (range, $6-14$ years old). On admission, the six patients had symptoms and signs of pneumonia, including fever, cough, and abnormal breath sounds. Headache and vomiting were noted in two cases, and chest pain in one patient. The duration of fever prior to admission ranged from 3 to 15 days (mean: $7 \pm 4.15$ days). The average total duration of fever was $19.5 \pm 8.34$ days (range, 9-31 days) and the average time before LVX therapy was $10 \pm 2.97$ days (range, $7-13$ days). After LVX treatment, the time of fever was from 1 to 3 days in five cases and 11 days in one case. The average hospital stay was $22.83 \pm 8.77$ days in these children. The clinical characteristics of these patients are displayed in Table 1 .

\section{Laboratory findings}

All the patients had normal white blood cell (WBC) counts, while the neutrophil percentage increased. The initial mean WBC count was $(8.18 \pm 3.01) \times 10^{9} / \mathrm{L}$ (neutrophil percentage: $0.84 \% \pm 0.07 \%)$. C-reactive protein increased dramatically in five children, but it was normal in one case. The initial serum lactate dehydrogenase ranged from 449 to $2,020 \mathrm{mg} / \mathrm{L}$. Two children were diagnosed with liver dysfunction due to the increases in alanine aminotransferase. Hypoproteinemia was found in three cases, cerebrospinal fluid abnormality in one case. Results of PPD skin test, and bacterial cultures of sputum and respiratory syncytial viruses, influenza viruses, parainfluenza virus, and adenovirus showed negative. Sputum for MPDNA and serum for MP IgM antibody were positive in all cases and the MP-DNA load was up to $10^{7}$ copies/mL (Max: 
Table 1 Clinical characteristics of five children with MRMPP

\begin{tabular}{|c|c|c|c|c|c|c|}
\hline Characteristic & Patient 1 & Patient 2 & Patient 3 & Patient 4 & Patient 5 & Patient 6 \\
\hline Age (years) & 6 & 8 & 6 & 6 & 7 & 14 \\
\hline Symptoms & $\begin{array}{l}\text { Cough and } \\
\text { fever }\end{array}$ & $\begin{array}{l}\text { Cough, fever, headache, } \\
\text { vomiting, chest pain }\end{array}$ & $\begin{array}{l}\text { Cough and } \\
\text { fever }\end{array}$ & $\begin{array}{l}\text { Cough and } \\
\text { fever }\end{array}$ & $\begin{array}{l}\text { Cough and } \\
\text { fever }\end{array}$ & $\begin{array}{c}\text { Cough, fever, } \\
\text { headache vomiting }\end{array}$ \\
\hline Before LVX treatment (day) & 7 & 12 & 8 & 13 & 13 & 7 \\
\hline After LVX treatment (day) & 1 & 2 & 2 & 3 & 11 & 2 \\
\hline Total fever duration (day) & 11 & 20 & 25 & 21 & 31 & 9 \\
\hline Fever $\left(\operatorname{Tmax},{ }^{\circ} \mathrm{C}\right)$ & 40 & 39.5 & 40 & 40.1 & 40.8 & 39 \\
\hline
\end{tabular}

MRMPP, macrolide-resistant Mycoplasma pneumoniae pneumonia; LVX, levofloxacin.

$10^{8}$ copies $/ \mathrm{mL}$ ). After ML treatment, the sputum MPDNA load was still at a high level and it decreased slowly or even increased again in six children. However, it decreased significantly after LVX therapy in five cases. In one child, the MP-DNA load was higher in sputum, throat swab, and pleural effusion specimen about $(2.7-2.86) \times 10^{7}$ copies $/ \mathrm{mL}$. Laboratory Findings in patients are shown in Table 2. The A2063G point mutation was found in the domain $\mathrm{V}$ of 23SrRNA gene of five cases (Figure 1). One case was not detected because the test was not carried out at that time.

\section{Radiographic findings}

In six children, the chest X-ray or computed tomography showed segmental or lobar alveolar infiltrates, and patchy clouding opacity in unilateral lung (right: $n=4$; left: $n=1$; both: $n=1$ ). Pulmonary consolidation was found in four cases, pulmonary atelectasis in two, and pleural effusion in five. The patients represented worsening lung shadow despite treatment with appropriate antimicrobials and adjunctive corticosteroid therapies (Figure 2). Electroencephalographic abnormalities were observed in three cases. One patient developed infection in both lungs and acute disseminated encephalomyelitis (ADEM) with abnormal brain magnetic resonance imaging (MRI) and occurred serious sequelae in Figure 3.

\section{Treatment}

Four children received cephalosporin therapy before admission and two children received azithromycin therapy or erythromycin treatment, but fever and cough remained. On admission, six children received intravenous azithromycin $(10 \mathrm{mg} / \mathrm{kg} / \mathrm{day})$. All the patients showed no clinical improvements or even clinical deteriorations demonstrated by chest radiography after ML and immunemodulators treatments. Thereafter, LVX therapy was initiated, and informed consent was obtained from their guardians before LVX therapy. LVX was administered intravenously at $7.5 \mathrm{mg} / \mathrm{kg} /$ time (twice daily) for 5-14 days. The body temperature returned to normal quickly in five cases at 1,2, 2, 2, and 3 days and one case at 11 days after LVX treatment, respectively. Glucocorticoids therapy was initiated in six children in whom intravenous methylprednisolone was given at $1-2 \mathrm{mg} / \mathrm{kg} / \mathrm{day}$ for 3-5 consecutive days in three cases, $10 \mathrm{mg} / \mathrm{kg} /$ day for three consecutive days in two patients, and intravenous dexamethasone was given at $0.1-0.25 \mathrm{mg} / \mathrm{kg} / \mathrm{day}$ for 7 days in one. After glucocorticoids therapy, the body temperature returned to normal, which remained for 2-3 days in three cases, but discontinuation of glucocorticoids treatment caused the increase in body temperature; three child had persistent fever after glucocorticoids treatment. Three children were treated with intravenous immunoglobulin (IVIG, $400 \mathrm{mg} / \mathrm{kg} / \mathrm{day}$ ) for three consecutive days.

\section{Discussion}

Since the ML-resistant MP was first reported in Japan in 2000, the number of MP isolates increased worldwide. The ML resistance mechanism is usually related to the point mutation of a specific locus in the domain $\mathrm{V}$ of $23 \mathrm{~S}$ rRNA 
Table 2 Laboratory findings in 6 children

\begin{tabular}{|c|c|c|c|c|c|c|c|c|c|}
\hline Patient & MP-DNA (sputum) & $\begin{array}{c}\text { MP-IgM } \\
\text { (serum) }\end{array}$ & MP-IgM (CSF) & ALT, IU/L & $\mathrm{LDH}, \mathrm{IU} / \mathrm{L}$ & ALB, g/L & WBC, $10^{9} / \mathrm{L}$ & CRP, mg/L & $\begin{array}{c}\text { Genetic } \\
\text { testing }\end{array}$ \\
\hline \multirow[t]{2}{*}{1} & A: $7 \times 10^{8}$ & + & NT & 47 & 637 & 38 & 6.3 & $>200$ & A2063G \\
\hline & C: $4.4 \times 10^{4}$ & & & & & & & & \\
\hline 2 & C: $1.61 \times 10^{8}$ & & & & & & & & \\
\hline \multirow[t]{2}{*}{3} & A: $9.1 \times 10^{5}$ & + & NT & 89 & 499 & 29.8 & 4.4 & $>160$ & A2063G \\
\hline & B: $1.61 \times 10^{8}$ & & & & & & & & \\
\hline 4 & C: $1.5 \times 10^{3}$ & & & & & & & & \\
\hline \multirow[t]{3}{*}{5} & A: - & + & NT & 153 & 2020 & 32 & 7.9 & 197 & A2063G \\
\hline & B: $4.9 \times 10^{7}$ & & & & & & & & \\
\hline & C: $2.5 \times 10^{5}$ & & & & & & & & \\
\hline \multirow[t]{3}{*}{6} & $A:-$ & + & + & 42 & 893 & 18 & 12.9 & $>200$ & NT \\
\hline & B: $2.09 \times 10^{7}$ & & & & & & & & \\
\hline & C: $1.8 \times 10^{5}$ & & & & & & & & \\
\hline
\end{tabular}

MP, Mycoplasma pneumoniae; CSF, cerebrospinal fluid; ALT, alanine aminotransferase; LDH, lactate dehydrogenase; ALB, albumin; WBC, white blood cell; CRP, C-reactive protein; A, before macrolide therapy; B, after macrolide therapy; C, after levofloxacin therapy.

gene (at positions 2063, 2064 and 2617), in which A-to-G transition is the most common mutation (11). In this report, A2063G mutation in the domain $\mathrm{V}$ of $23 \operatorname{Sr} R N A$ gene was found in five patients. Previous studies have shown that, compared with ML sensitive MPP patients, ML resistance MPP patients have longer total febrile period, longer median fever duration after ML therapy, longer median hospitalization duration, more extrapulmonary complications, and more severe clinical radiological manifestations (12). These were also confirmed in six children treated in our hospital. These children with ML resistance MPP showed persistent fever and radiographic progression after ML treatment.

Glucocorticoids have strong anti-inflammatory effect and immunosuppressive effect, and may be used for the treatment of severe MPP with favorable efficacy. However, the timing and dosage of glucocorticoids are still unclear in children. Usually, oral or intravenous glucocorticoids are administered at $1-2 \mathrm{mg} / \mathrm{kg} /$ day for $3-7$ days (13). IVIG plays an important role in preventing the disease from progressing into severe inflammation by blocking protein cascade pathways in the inflammation (14). IVIG also has the immune-modulatory activity, which has involvement of $\mathrm{Fc}$ receptor blockage, neutralization of etiologic agents and suppression of activated immune cells (15). Some investigators have shown that IVIG can be used to treat extrapulmonary manifestations of MP infection, especially the nervous system diseases. However, there is still a lack of high quality evidence about the therapeutic efficacy of IVIG alone, and more randomized, controlled studies are needed (13). In this study, all children showed persistent fever and were non-responsive to the combined treatment with glucocorticoids and IVIG.

Fluoroquinolones such as ciprofloxacin, moxifloxacin and LVX combined with glucocorticoids have been shown to be effective for the treatment of ML resistant MPP. However, 


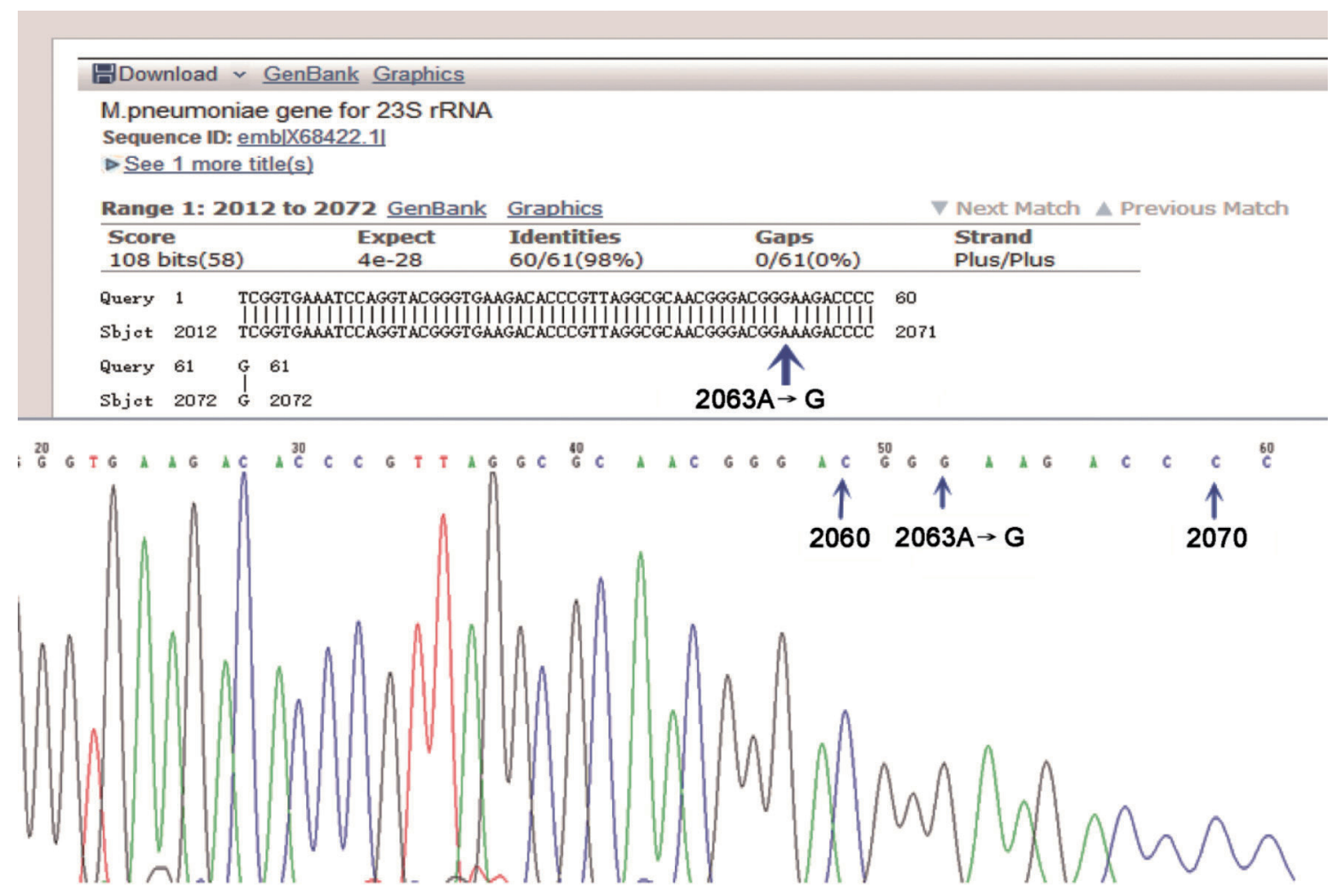

Figure 1 The A2063G point mutation in the domain $\mathrm{V}$ of $23 \operatorname{Sr} R N A$ gene.
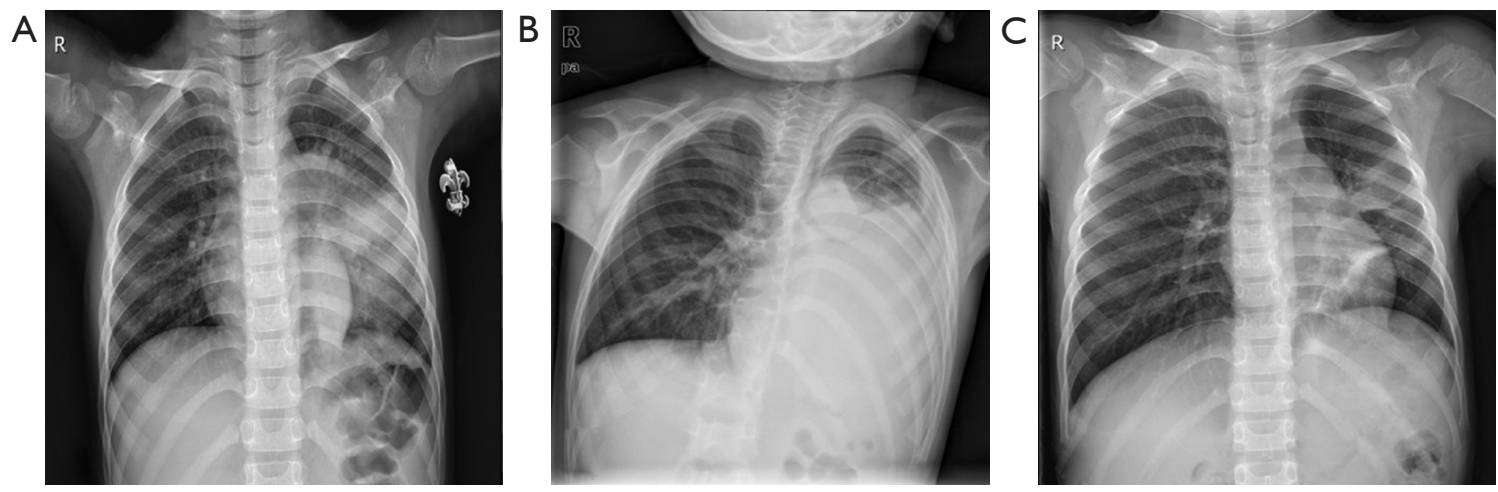

Figure 2 The lung shadow in X-ray. (A) A 7-year-old boy (day 0) with proved left lung infection; (B) presenting chest X-ray (day 6) demonstrates worsening lung shadow and left pleural effusion; (C) the X-ray shows (day 23) apparently absorption.

no study has been reported to use fluoroquinolones (such as LVX) alone in the treatment of ML resistant MPP. In the present report, six children showed persistent fever and were non-responsive to $\mathrm{ML}$ and immune-modulators therapy. However, after initiation of LVX treatment, the fever was improved within 3 days in five cases, suggesting its effectiveness. Recently, Japanese investigators reported that minocycline or tosufloxacin was more effective than ML in the treatment of ML resistant MPP (16). When ML therapy failed, minocycline or doxycycline could be used as the first-line treatment for ML resistant MPP in children aged $>8$ years old, and fluoroquinolones could be applied in children aged $<8$ years old (16). LVX is the third generation of fluoroquinolone antibiotic with broad-spectrum 

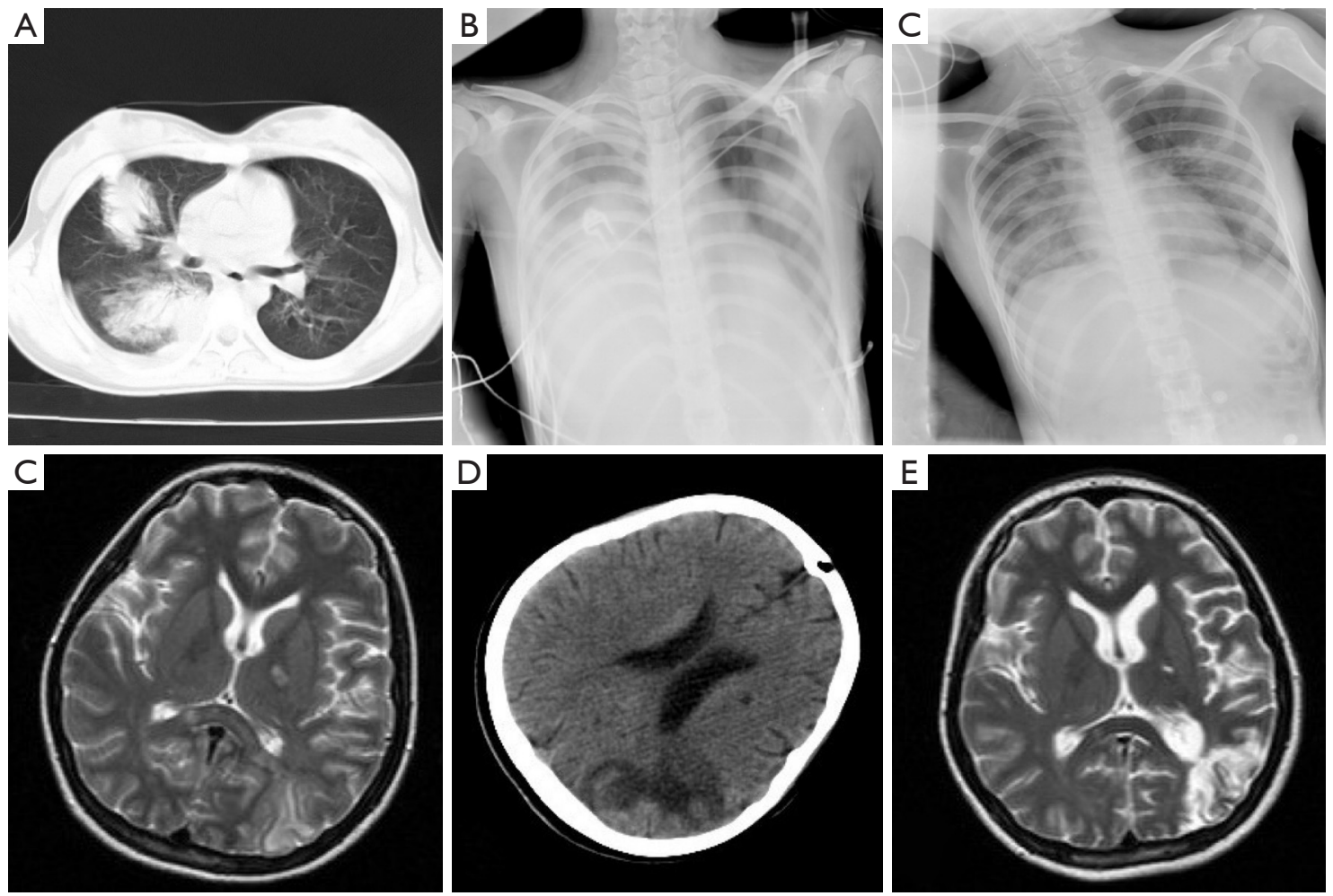

Figure 3 The iconography in both lung and brain. (A) A 14-year-old girl (day 0) with both lungs infection in chest CT; (B) chest X-ray (day 3) demonstrates worsening lung shadow and right pleural effusion; (C) X-ray shows (day 14) significantly absorption; (D) head MRI (14 day) showed multiple abnormal signal lesions considering infection with hemorrhage; (E) reexamination of the head CT (30 day) showed that the left occipital region and bilateral cerebellar multiple lesions and hemorrhage tending to soften; (F) MRI (3 month) demonstrated multiple lesions with bleeding and softening in left cerebral hemisphere and corpus callosum.

bactericidal activity against Gram-positive and Gramnegative bacteria as well as atypical respiratory pathogens and high bactericidal activity against MP in vitro and in vivo $(17,18)$. Moreover, it has good tissue penetration and is well tolerated $(17,18)$. In the study of Bradley et al. (19), the clinical cure rate of CAP (MP is the main pathogen) was $94.3 \%$ (382 out of 405) in LVX-treated children, and therefore they speculated that LVX was as tolerated and effective as standard-of-care antibiotics for the treatment of CAP in children. In the 2011 Clinical Practice Guideline for CAP in Children by the Pediatric Infectious Diseases Society and the Infectious Diseases Society of America, LVX is recommended for the treatment of severe MPP (20). Preclinical studies have reported that fluoroquinolones used in juvenile animals can induce articular cartilage damage, but the use of fluoroquinolones in some children failed to show any significant side effects affecting growth and development, or show transient, and curable adverse effects.

The decreased MP DNA copies in the sputum were well correlated with clinical symptoms and therapeutic efficacy of antibiotics. In the study of Okada et al. (16), the MP-DNA copies in throat swabs decreased from $10^{6}$ copies $/ \mathrm{mL}$ to $2 \times 10^{4}$ copies $/ \mathrm{mL}$ after 3 -day treatment and $1.5 \times 10^{4}$ copies $/ \mathrm{mL}$ after 5 -day treatment in patients receiving tosufloxacin treatment. Kawai et al. (5) reported, in patients with MRMPP, the decrease of MP load after 48-96 h treatment was more rapid in tosufloxacin treated patients than in azithromycin treated patients. Among MRMPP patients, the rate of MP eradication in the nasopharynx at the end of treatment was $34 \%$ in the azithromycin treated patients and $59 \%$ in the tosufloxacin-treated patients; defervescence within $48 \mathrm{~h}$ after the initiation of antibiotics treatment was observed in $41 \%$ of azithromycin treated patients and $69 \%$ of tosufloxacin-treated patients; the average febrile period after antibiotic treatment was lower in the tosufloxacin group than in the ML group. In our report, the six children with severe infection showed high MP-DNA load, and the MP-DNA copies decreased slowly 
or even increased after ML treatment. However, after LVX treatment, the MP-DNA copies decreased significantly. This suggests that LVX may achieve a good therapeutic efficacy for MRMPP when it is non-responsive to ML. Moreover, the decrease rate of sputum MP-DNA was related to the clinical manifestations and therapeutic efficacy. Cheng et al. reported (21) a close relationship between MP load of bronchoalveolar lavage fluid and clinical characteristics in children with MP pneumonia, which was that high MP load had more severe disease. Our findings were consistent with above findings. MP-DNA load was not high initially in one case, but after ML treatment it increased to $1.61 \times 10^{8} / \mathrm{L}$. Thus, whether the use of ML contributes to the development of ML-resistant strains remains to be further elucidated. In our report, four children were followed up by the measurement of sputum MP-DNA via PCR, of whom three patients eventually became PCR negative at 1,1 , and 3.5 months after disease onset, respectively, but one patient remained positive for six weeks. One patient lost to followup. Nilsson et al. reported (22) MP-DNA usually remained detectable on PCR in throat swabs for several weeks after symptom onset and could persist for months with a slow decline in bacterial load over time. In the present report, all the patients eventually became PCR negative except for one who were PCR-positive for seven months. Spuesens et al. reported (23) the MP-DNA load of the respiratory tract determined by PCR in 22 symptomatic children with MP, and found 19 children became negative after one month, and three children were negative at three months. Thus, a positive PCR test for MP-DNA is sometimes difficult to distinguish between MP re-infection and asymptomatic carriage, and the value of MP-DNA measurement should be further investigated in more studies.

ADEM following MP infection is a rare condition. It should be in the differential diagnosis whenever respiratory symptoms are followed by neurological manifestations and encephalopathic signs (24). Brain MRI is extremely useful to establish a diagnosis of ADEM; with better visualization of the demyelinating lesions (25). In this study, one patient developed ADEM with abnormal brain MRI and occurred serious sequelae. The clinical presentations of patients were unusual in four aspects in this study. First, in this study, there were six children with proved RMPP with worsening lung shadow despite treatment with appropriate antimicrobials and corticosteroid. Second, attention should be paid to children who was older than six years old, had fever more than one week and worse imaging findings. It is necessary to dynamically monitor blood routine, chest X-ray, or computed tomography three to five days after admission. Third, LVX may be considered in critical cases of RMPP and MRMPP with age greater than seven years. Fourth, lung infections were well absorbed around one month, but prognosis may be poor when brain imaging is abnormal in children with MPP.

\section{Conclusions}

In conclusion, LVX may be preferred for the treatment of MRMPP when the therapeutic efficacy of ML and immunemodulators is unsatisfactory. Besides, the decreased MPDNA copies in the sputum are well correlated with clinical characteristics and therapeutic efficacy of antibiotics. Our results suggest that LVX may be promising antimicrobial agent for the treatment of ML-resistant MP infection. We found that RMPP can be gradually repaired with effective treatment of quinolone, but it can damage the nervous system and lead to severe invasion of MP into brain tissues. However, the sample size was small, and more clinical studies are needed to confirm our findings. Pediatricians should improve the early recognition of MRMPP, which is important for its prognosis.

\section{Acknowledgments}

None.

\section{Footnote}

Conflicts of Interest: The authors have no conflicts of interest to declare.

Ethical Statement: The authors are accountable for all aspects of the work in ensuring that questions related to the accuracy or integrity of any part of the work are appropriately investigated and resolved. This study was approved by the Ethics Committee of Second Affiliated \& Yuying Children's Hospital (No. LCKY2019-105).

\section{References}

1. Meyer Sauteur PM, Jacobs BC, Spuesens EB, et al. Antibody responses to Mycoplasma pneumoniae: role in pathogenesis and diagnosis of encephalitis? PLoS Pathog 2014;10:e1003983.

2. Shah SS, Test M, Sheffler-Collins S, et al. Macrolide therapy and outcomes in a multicenter cohort of children 
hospitalized with Mycoplasma pneumoniae pneumonia. J Hosp Med 2012;7:311-7.

3. Hawkins S, Rausch CM, McCanta AC. Constrictive pericarditis secondary to infection with Mycoplasma pneumoniae. Curr Opin Pediatr 2011;23:126-9.

4. Zhao F, Liu G, Wu J, et al. Surveillance of macrolideresistant Mycoplasma pneumoniae in Beijing, China, from 2008 to 2012. Antimicrob Agents Chemother 2013;57:1521-3.

5. Kawai Y, Miyashita N, Kubo M, et al. Therapeutic efficacy of macrolides, minocycline, and tosufloxacin against macrolide-resistant Mycoplasma pneumoniae pneumonia in pediatric patients. Antimicrob Agents Chemother 2013;57:2252-8.

6. Esposito S, Tagliabue C, Bosis S, et al. Levofloxacin for the treatment of Mycoplasma pneumoniae-associated meningoencephalitis in childhood. Int J Antimicrob Agents 2011;37:472-5.

7. Lu A, Wang L, Zhang X, et al. Combined treatment for child refractory Mycoplasma pneumoniae pneumonia with ciprofloxacin and glucocorticoid. Pediatr Pulmonol 2011;46:1093-7.

8. Shen Y, Zhang J, Hu Y, et al. Combination therapy with immune-modulators and moxifloxacin on fulminant macrolide-resistant Mycoplasma pneumoniae infection: A case report. Pediatr Pulmonol 2013;48:519-22.

9. Saunders M, Gorelick MH. Nelson textbook of pediatrics. 19th ed. Philadelphia: Saunders Elsevier, 2011.

10. Subspecialty Group of Respiratory Diseases TSoP, Chinese Medical Association. Expert consensus on diagnosis and treatment of Mycoplasma pneumoniae Pneumonia in children (2015). Clin J Appl Clin Pediatr 2015:1304-8.

11. Liu X, Jiang Y, Chen X, et al. Drug resistance mechanisms of Mycoplasma pneumoniae to macrolide antibiotics. Biomed Res Int 2014;2014:320801.

12. Zhou Y, Zhang Y, Sheng Y, et al. More complications occur in macrolide-resistant than in macrolide-sensitive Mycoplasma pneumoniae pneumonia. Antimicrob Agents Chemother 2014;58:1034-8.

13. Liu H, Lu Q, Hong J, Liu E. A systematic review of the therapy for Mycoplasma pneumoniae infections in children. Zhonghua Er Ke Za Zhi 2016;54:111-8.

14. Tha-In T, Bayry J, Metselaar HJ, et al. Modulation of the cellular immune system by intravenous immunoglobulin. Trends Immunol 2008;29:608-15.

15. Lee KY, Lee JS. Immunoglobulin G has a role for systemic protein modulation in vivo: a new concept of protein homeostasis. Med Hypotheses 2006;67:848-55.

16. Okada T, Morozumi M, Tajima T, et al. Rapid effectiveness of minocycline or doxycycline against macrolide-resistant Mycoplasma pneumoniae infection in a 2011 outbreak among Japanese children. Clin Infect Dis 2012;55:1642-9.

17. Choi SH, Kim EY, Kim YJ. Systemic use of fluoroquinolone in children. Korean J Pediatr 2013;56:196-201.

18. Velissariou IM. The use of fluoroquinolones in children: recent advances. Expert Rev Anti Infect Ther 2006;4:853-60.

19. Bradley JS, Arguedas A, Blumer JL, et al. Comparative study of levofloxacin in the treatment of children with community-acquired pneumonia. Pediatr Infect Dis J 2007;26:868-78.

20. Bradley JS, Byington CL, Shah SS, et al. The management of community-acquired pneumonia in infants and children older than 3 months of age: clinical practice guidelines by the Pediatric Infectious Diseases Society and the Infectious Diseases Society of America. Clin Infect Dis 2011;53:e25-76.

21. Cheng YG, Li SX, Li XJ, et al. Relationship between bronchoalveolar lavage mycoplasma load and clinical characteristics in children with Mycoplasma pneumoniae pneumonia. Zhonghua Er Ke Za Zhi 2013;51:736-40.

22. Nilsson AC, Bjorkman P, Persson K. Polymerase chain reaction is superior to serology for the diagnosis of acute Mycoplasma pneumoniae infection and reveals a high rate of persistent infection. BMC Microbiol 2008;8:93.

23. Spuesens EB, Fraaij PL, Visser EG, et al. Carriage of Mycoplasma pneumoniae in the upper respiratory tract of symptomatic and asymptomatic children: an observational study. PLoS Med 2013;10:e1001444.

24. Cheng CC, Fan HC, Chi CS, et al. Acute Disseminated Encephalomyelitis Secondary to Mycoplasma pneumoniae Infection Mimicking Extrapyramidal Symptoms: A Case Report. J Pediatr Neurol 2018;16:015-20.

25. Laila A, El-Lababidi RM, Hisham M, et al. A case of acute disseminated encephalomyelitis following Mycoplasma pneumoniae infection. IDCases 2018;12:41-3.

Cite this article as: Lin M, Shi L, Huang A, Liang D, Ge L, Jin Y. Efficacy of levofloxacin on macrolide-unresponsive and corticosteroid-resistant refractory Mycoplasma pneumoniae pneumonia in children. Ann Palliat Med 2019;8(5):632-639. doi: 10.21037/apm.2019.10.05 